\title{
CONFLICTOS SOCIOAMBIENTALES: ENTRE LA LEGITIMIDAD NORMATIVA Y LAS LEGITIMIDADES SOCIALES. CASO MINA LA COLOSA, CAJAMARCA (TOLIMA, COLOMBIA). ${ }^{1}$
}

\author{
Claudia Alexandra Munévar Quintero \\ Luisa Fernanda González Londoño (1D ${ }^{3}$ \\ Anyela Andrea Henao Londoño (D) 4
}

Recibido el 9 de julio de 2015, aprobado el 4 de marzo de 2016 y actualizado el 16 de diciembre de 2016

DOI: 10.17151/luaz.2017.44.10

\section{RESUMEN}

El presente artículo tiene como marco de análisis la descripción de un conflicto socioambiental, presentado en razón del proyecto minero adelantado en la mina La Colosa del municipio de Cajamarca (Tolima) en Colombia. En primer lugar, se conceptualiza sobre las diferentes posiciones teóricas del conflicto socioambiental a partir de teorías jurídicas, sociales y ambientales. Se analizan las diferentes dimensiones sociales, económicas y políticas del conflicto y se puntualiza en la descripción del caso objeto de estudio. En segundo lugar, se problematiza en torno a la legitimidad de las normas y las legitimidades sociales, a partir de lo analizado en el caso estudiado. Por último, se concluye que el alcance de la legitimidad perseguido por las normas y por la institucionalidad representada en el Estado no se agota en el simple cumplimiento de las normas. La legitimidad se materializa a través de la aceptación por parte de los asociados y de su correspondencia con las percepciones sociales.

\section{PALABRAS CLAVE}

Conflicto socioambiental, legitimación normativa, legitimidades sociales.

\section{SOCIO-ENVIRONMENTAL CONFLICTS: BETWEEN NORMATIVE LEGITIMACY, AND SOCIAL LEGITIMACIES. THE COLOSA MINE IN CAJAMARCA TOLIMA, COLOMBIA) CASE STUDY.}

\section{ABSTRACT}

The description of a socio-environmental conflict serves as the analytical framework of this article. It is a mining project carried out in The Colosa Mine located in the municipality of Cajamarca (Tolima) in Colombia. First, the different theoretical positions concerning the socio-environmental conflict from legal, social and environmental theories are conceptualized. Different social, economic and political dimensions of the conflict are also analyzed focusing on the description of the case under study. Secondly, relevant problematic issues about the legitimacy of certain norms and social legitimacies emerge from the analyzed case study. Finally, it is concluded that the scope of the legitimacy pursued by the rules as well as the institutional aspects representing the State go beyond the simple compliance of the norms. Legitimacy is achieved through the acceptance on the part of the partners and its correspondence with social perceptions. 


\section{KEY WORDS}

Socio-environmental conflict, legitimacy of rules, social legitimacies.

\section{INTRODUCCIÓN}

El significado de conflicto ha sido abordado desde diferentes teorías, ciencias y disciplinas, generalizando su concepto a confrontaciones y contradicciones entre grupos a partir de hechos, acciones y percepciones. A partir de esta significación, el artículo conceptualizará teóricamente el alcance del conflicto desde una mirada sociojurídica y ambiental, presentado las diferentes concepciones y teorías que definen este concepto. De igual forma, se contextualizará su noción a partir del estudio de caso de un conflicto socioambiental, descrito a partir del proyecto minero en el municipio de Cajamarca (Tolima).

A partir de estas divergencias se cuestionará el alcance del conflicto no solo en las esferas sociales, sino también frente al alcance de la legitimidad de las normas en términos materiales y sociales. Se discutirán las diferentes formas como se legitima el poder de dominación de las estructuras normativas y del Estado y de cómo, a partir de ellas, se generan las confrontaciones que dan lugar al conflicto.

\section{CONCEPTUALIZACIÓN DEL CONFLICTO SOCIOAMBIENTAL}

Diferentes posiciones teóricas se han aproximado hacia la definición del conflicto. La primera de ellas, desde una perspectiva atribuible a los científicos sociales a partir de la construcción de una teoría del conflicto. Silva (2008) presenta las variantes históricas de las teorías conflictualistas desde las posturas de Marx y la teoría sociológica liberal del conflicto. Sobre el primero, menciona que para Marx la unidad básica de análisis para mencionar el conflicto son las clases sociales, las cuales inician con luchas por los intereses enfrentados con las estructuras de poder. Sin embargo, para la teoría sociológica liberal, la unidad de clase social es general, puesto que no todos los conflictos tienen referente en ella (Silva, 2008, p. 31). En este sentido, esta teoría emplea la categoría de grupo social, entendiendo que existen otras partes asociadas al conflicto, diferentes a las clases sociales.

El conflicto, desde la perspectiva social, se reconoce como un proceso inherente a las interacciones sociales. Coser (1961, p. 42) establece: "El conflicto social siempre denota una interacción social", producto de "una situación de divergencia social, es decir, de una relación contradictoria (disputa) que sostienen personas o grupos sociales separados al poseer intereses y/o valores diferentes" (Silva, 2008, p. 36). En este sentido, se puede afirmar que el conflicto hace parte de las construcciones ${ }^{5} y$ trasformaciones sociales y humanas, va implícito en las relaciones del hombre y, como lo establece Marx, ha contribuido a los cambios y progresos sociales.

Esta perceptiva del conflicto social permite identificar tres aspectos esenciales en la conformación del conflicto: hace parte de las relaciones sociales, lo constituyen partes o sujetos representados por individuos o instituciones, y exige la existencia de un objeto de confrontación. Ahora bien, cada una de estas características da lugar a la composición de diferentes clases de conflictos, según el tipo de relaciones e interacciones sociales, la identidad de las partes y el objeto que las confronta. 
El objeto de confrontación define la naturaleza del conflicto, en el sentido que materializa la concepción de disputa e individualiza las partes. Cuando el objeto de disputa lo conforman aspectos relacionados con el ambiente o los recursos naturales se conocen como conflictos ecológicos o ambientales. Al respecto, Pérez, Zárate \& Turbay (2011, p. 93) establecen: "Los conflictos resultantes de la relación entre el ser humano y la naturaleza se conocen como conflictos ambientales o socio-ambientales, referenciados en la literatura también como conflictos inducidos por el ambiente, ecológico-distributivos o de contenido ambiental". No obstante, cada definición aporta una tesis con alcances y sentidos diferentes. Desde la perspectiva ecológica, autores como García (2014a, p. 11) hacen relación, entre otros aspectos, a conflictos "relacionados con la inequidad frente al acceso de recursos por parte de la población local". Asimismo, Martínez-Alier (2006) se refieren a conflictos ecológico-distributivos como aquellos que si bien se encuentran por fuera del mercado, la economía genera incidencia en los resultados de dichos conflictos.

Por otro parte, con referencia a los conflictos ambientales, Pérez et al. (2011, p. 93) atribuyen la definición de conflicto ambiental al "tema de disponibilidad y acceso a los recursos naturales". No obstante, se considera que la compresión netamente ecológica o asociada a recursos naturales resulta reduccionista frente a la definición del conflicto por causas ambientales, por cuanto estos no se forman solo por la fijación de los precios del mercado o por el agotamiento de determinado recurso, sino también por el valor que cada una de las partes le otorgan al objeto que está en confrontación. Estos conflictos, denominados socioambientales ${ }^{6}$, involucran aspectos ambientales, pero también culturales, ideológicos e identitarios que asocian relaciones y correspondencias con un espacio territorial y social de las partes que disputan un objeto de poder. Cuando se habla de un objeto de poder debe entenderse que este no solo depende de concepciones económicas, sino también sociales y culturales de las partes en conflicto. Para una institución el objeto de poder puede ser un factor económico, para una comunidad puede ser su territorio y las identidades construidas en él. Estas características aplican a diferentes escenarios de conflictos y constituyen un marco descriptivo de contextos sociales, donde se exponen estas categorizaciones de conflictos y donde se identifican diferentes actores en confrontación.

\section{CONTEXTUALIZACIÓN DE UN CONFLICTO SOCIOAMBIENTAL: CASO MINA LA COLOSA, CAJAMARCA (TOLIMA)}

El contexto del presente conflicto se encuentra ubicado en el municipio de Cajamarca (Tolima), en donde se adelanta el denominado proyecto minero "La Colosa", ejecutado por la empresa surafricana AngloGold Ashanti. El Estado colombiano concedió a la multinacional título de exploración minera, el cual se ha desarrollado en dos fases: prefactibilidad, entre los años 2010-2016 y factibilidad, entre los años 2017-2018. Según informes presentados por la sala de prensa de la multinacional minera, el proceso de licenciamiento estaría para el año 2018, culminando el proyecto minero para el año $2022^{7}$.

El municipio de Cajamarca se caracteriza por su vocación agrícola y por estas características, Cajamarca es denominado como la "despensa agrícola de Colombia". ${ }^{8}$ Dentro de las políticas y normatividades del municipio, se tiene que dentro del Plan de Desarrollo del Municipio "Para volver a creer" (2012-2015), se considera como factor fundamental del desarrollo y la sostenibilidad del territorio el fortalecimiento y la competitividad del sector agropecuario, toda vez que esta apuesta generaría no solo el mejoramiento del PIB municipal, sino también fortalecería la generación de empleo y consumo. De igual forma, como propuesta de consolidación de la identidad cultural del municipio, el artículo 238 del Esquema de Ordenamiento 
Territorial, estableció como proyectos prioritarios "reforzar la convicción colectiva de que Cajamarca es la Despensa Agrícola del Departamento y del país".

La realización de un proyecto minero contrastaría con la identidad histórica de Cajamarca, que se ha caracterizado como un municipio de vocación agrícola y no minera. Características que materializan un conflicto socioambiental en torno a la frontera agrícola con la minera, y que institucionalizan el conflicto socioambiental desde tres de las cuatro dimensiones planteadas por Fontaine (2004, p. 512): "La institucionalización de los conflictos socio-ambientales depende del entendimiento de las interacciones entre los actores en estas cuatro dimensiones": social, "que se origina en la sociedad civil con la formación de movimientos sociales ambientalistas, campesinos [...]"; político, "a través de su institucionalización [...]; y económica, "debida a la presión de la deuda externa y a los procesos de integración regional, y una dimensión ética del punto de vista de la consolidación de los procesos de democratización y de paz".

Respecto a la dimensión social, existen unas partes y unos sujetos representados por la sociedad civil; algunos de ellos se han constituido e identificado como movimiento sociales, cuya lucha y confrontación se enmarcan en el plano de la defensa del territorio, identidades, culturas, relaciones y todas las dimensiones de alcance estructural o interaccionista. Desde la entrada de la multinacional ha habido oposiciones por parte de la comunidad y dicha dimensión social se ha materializado por medio de movimientos sociales. Líderes activos de la comunidad han manifestado un proceso inicialmente de sensibilización a la comunidad, generando marchas, encuentros, audiencias públicas y convocatorias a consultas populares. Todos estos actos son manifestaciones de luchas por la defensa de sus territorios. Como lo define Silva $(2008$, p. 35$)$ : "[...] la conflictividad social penetra todos los campos de la vida sin excepción y [...], alcanza tanto la dimensión macrosocial como la microsocial de la vida social".

En segundo lugar, la dimensión económica se materializa de forma más visible en la contienda que envuelve el conflicto. Para el caso objeto de estudio, una de las confrontaciones que abarca la dimensión económica radica en las operaciones de la empresa AngloGold Ashanti. Esta compañía productora de oro tiene sus operaciones actualmente en cuatro continentes y constituye una multinacional de alto impacto financiero, que ofrece alternativas económicas para el municipio y sus pobladores, ofertadas en regalías, generación de empleos y actividades alternas como el comercio, hotelería y turismo, que son externalidades positivas del proyecto minero. No obstante, también se generan otro tipo de externalidades negativas como el deterioro social y ambiental con problemáticas como la informalidad del empleo, encarecimiento de los costos de vida, violencia, cambio de uso del suelo, degradación ambiental, entre otro tipo de externalidades que, como lo manifiestan Martínez \& Roca (2001, p. 21), "dan lugar a movimientos de resistencia que utilizan distintos lenguajes sociales".

Por lo anterior, la explotación minera se convierte en una alternativa económica importante para algunos sectores comunitarios y políticos, pero a su vez presupone un detrimento en la vocación cultural, social y económica del municipio. Estos cambios de configuraciones evidencian externalidades ${ }^{9}$ y confrontaciones frente a las valoraciones, toda vez que unas pueden ser de tipo económico y otras de tipo simbólico. Como lo establece Garay (2013, p. 14): "[...] estos conflictos locales y globales se expresan a través de distintos lenguajes de valoración bajo cosmologías divergentes [...]. Se trata entonces [...] de diversos sistemas de valoración en tensión y disputa: la valoración del mercado y la valoración simbólica/cultural/identitaria". 
Las actividades productivas de esta región del Tolima relacionan las identidades de la población con el reconocimiento al campo y a la tierra. Como se estableció anteriormente, dentro de las políticas establecidas en el Esquema de Ordenamiento Territorial del municipio de Cajamarca se propone posicionar al municipio como productor agropecuario, con el fin de mejorar la competitividad en el contexto regional y nacional ${ }^{10}$. Estas dobles vías económicas desatan contraposiciones de visiones y percepciones frente a la vocación económica del municipio y generan conflictos en cara a esta dimensión.

Por último lugar, respecto a la dimensión política, existen, como lo manifiesta Leff (2003, p. 20), procesos extraeconómicos como los ecológicos y políticos "que desplaza[n] a la racionalidad económica al campo de la economía política”. Estos procesos convierten a la naturaleza en un "referente político, no solo de una política de Estado para la conservación de las bases naturales de sustentabilidad del planeta, sino como objeto de disputa y apropiación social" (Leff, 2003, p. 22).

En este sentido, desde esta dimensión se pueden enunciar dos perspectivas que la definen. Por un lado, la institucionalización (Fontaine, 2004); y por el otro, los postulados de poder, a lo que Silva $(2008$, p. 30$)$, establece que: "las teorías sociológicas del conflicto son, por excelencia, teorías de sociología política, ya que el tema de poder ocupa un lugar principalísimo entre sus postulados". Dentro de las muchas significaciones de poder, se puede entender en términos generales "(...) la posibilidad de que una persona, o varias, realicen su propia voluntad en una acción en común, aun en contra la oposición de otros participantes en la acción" (Weber, 2006, p. 45). Esta voluntad materializa esa institucionalidad, que respaldadada por factores de poder, actúa como parte del conflicto, pero en escenarios que, en palabras de Weber (1944), lo hace bajo estructuras de dominación, legitimadas por aspectos normativos o legales.

Explicando la lógica de la dimensión política y de los poderes de dominación visibilizados en este caso, la AngloGold Ashanti inicia su fase de exploración por medio de un proceso legal, sustentado y respaldado en el ordenamiento jurídico colombiano, a través de la Ley 685 de 2001: "Por la cual se expide el Código de Minas y se dictan otras disposiciones". Allí se encuentran los lineamientos y criterios necesarios para la obtención de títulos mineros en Colombia. De igual forma, en cumplimiento a lo establecido en el artículo 80 de la Constitución Política ${ }^{11}$, el Estado colombiano, a través de la autoridad ambiental, tiene la responsabilidad de planificar la administración de los recursos naturales, con el fin de contribuir a sus sostenibilidad. En este sentido, es el Estado el legitimado legalmente para otorgar los permisos, títulos o licencias en toda fase de exploración o explotación.

En este sentido, este proyecto de exploración se legitima desde dos estructuras: una normativa y otra institucional. Normativa, toda vez que desde el orden jurídico se autoriza la explotación del recurso con base en lineamientos y políticas estatales, las cuales plantea el Estado a partir de sus Planes de Desarrollo. Para el caso de los proyectos mineros, el Plan Nacional de Desarrollo 2010-2014, estableció las actividades que impulsarán económicamente el desarrollo del país bajo la denominación de locomotoras de crecimiento ${ }^{12}$, dentro de las cuales se encuentra el desarrollo minero-energético, identificada como la cuarta locomotora ${ }^{13}$, la cual, según lo establecido por el Plan Nacional de Desarrollo 2010-2014 (p. 208): "es innegable que este sector es y será en los próximos años uno de los ejes centrales de la economía colombiana".

Respecto a la segunda estructura, esto es, la institucional, la legitimidad normativa descansa en una autoridad legal. Una de las afirmaciones y defensa de la AngloGold, 
es el otorgamiento de permisos y vigilancia por parte de las autoridades ambientales ${ }^{14}$. Al respecto, Weber (1944, p. 172) establece que la dominación legítima "descansa en la creencia de la legalidad de ordenaciones estatuidas y de los derechos de mando de los llamados por esas ordenaciones a ejercer la autoridad (autoridad legal)".

Esta legitimación identifica más sujetos en las partes de confrontación. No solo obedece a los sujetos descritos en la primera dimensión social, donde se mencionan los miembros de la sociedad civil o movimientos sociales, sino que además estas estructuras de dominación o poder visibilizan otros actores como la empresa y el Estado. El primero, como el sujeto que opera el proyecto y el segundo, porque legitima la operación del mismo. En sentido, las partes aunque representan dos escenarios de confrontación, la constituyen tres actores, que para el caso en concreto, tanto la empresa, como el Estado, (representando el segundo escenario de confrontación a través de dos actores), manifiestan el poder a través de la ley y la institucionalidad. Weber (2006, p. 45), establece:

Hay ley cuando existe la posibilidad de mantener un orden mediante un conjunto específico de hombres que aplicarán la coacción física o psíquica con el objeto de lograr una aceptación del orden o de sancionar una transgresión. El reparto de poder económico y de otra clase, depende directamente del ordenamiento legal existente en la comunidad. Esto es aplicable no sólo al Estado sino a todos los órdenes legales.

En este sentido, se pueden interpretar dos tipos de coacciones. Coacción física: la obligatoriedad de algo, so pena de una sanción como la prisión; o la coacción psíquica, que podría considerarla también como una coacción simbólica ${ }^{15}$. Al respecto García (2014b, p. 92):

Las normas jurídicas también pueden ser símbolos dirigidos a la representación. En este caso, su fuerza radica en el impacto mental que produce un discurso legal con capacidad para establecer la diferencia entre lo lícito y lo ilícito, lo justo o lo injusto, lo verdadero y lo falso y entendido como parte fundamental de instituciones político-jurídicas legítimas.

En el contexto de los conflictos socioambientales, hay poder del Estado cuando a través de la legitimidad de las normas y autoridades ambientales se autorizan e imponen los proyectos, así la comunidad se oponga. Este evento podría materializarse como una coacción psíquica, toda vez que a través del permiso que otorga el título de exploración o la licencia de explotación se genera ese impacto mental psíquico de legalidad y, por ende, de legitimidad.

\section{ENTRE LA LEGITIMIDAD NORMATIVA Y LAS LEGITIMIDADES SOCIALES}

Podría afirmarse en un primer momento que el actuar de una de las partes en conflicto (Empresa y Estado), goza de una presunción de legitimidad desde la perspectiva legal, mas no social. La legitimidad social se sustenta en la aceptación de las normas; aceptación que puede obedecer a razones políticas o ideológicas que, en palabras de Alarcón (2011), no solo depende de un mandato legal. En este sentido, la mera existencia de las normas no es una condición de legitimidad, toda vez que se requiere una aceptación por parte de la comunidad. Al respecto, Durán (1997, p. 33), establece:

No se puede omitir que el derecho no se agota en el derecho positivo, y por ello es obligado un acercamiento al derecho desde diferentes perspectivas: no solo desde el derecho que se aplica, sino también desde la legitimidad del 
contenido de las normas jurídicas; y también desde la aceptación de dichas normas por parte de los miembros del grupo.

Son los criterios de legitimación los que debe perseguir el derecho y esta concepción de legitimidad no solo radica en las definiciones otorgadas por la filosofía o sociología del derecho. La legitimidad de las normas y de las instituciones que las emiten también se ubican en los juicios de percepción de los asociados, en el sentido que esta legitimidad sea percibida, es decir, que los valores que pretenden alcanzarse no entren en conflicto o contradicciones. Como lo menciona García (2014b, p. 64): "casi nadie duda de los valores cuando se miran de manera aislada, uno por uno [...]. El problema se presenta cuando dos o más valores entran en conflicto en un caso en concreto". Así, para el Estado o la empresa el área a intervenir es considerada como un recurso de potencial aprovechamiento o explotación, pero para las comunidades ese territorio es más que un espacio físico o material, es un espacio simbólico donde se desarrollan sus formas de vida, ideologías e identidades.

Esta confrontación de valores y apreciaciones sobre el territorio, expresa el conflicto socioambiental descrito en el municipio de Cajamarca y se explica, entre otras razones, por las contradicciones y confrontaciones entre los marcos normativos que dan lugar al conflicto. Por un lado, el Estado, que dentro de las estipulaciones constitucionales y legales, atiende a sus políticas de desarrollo, cuyos propósitos y finalidades, entre otros, se enfocan en la explotación minera y en la atracción de capital extranjero para dicho fin. Este capital extranjero se materializa con la entrada de la multinacional AngloGold Ashanti, empresa cuyo proceder se legitima dentro del marco normativo que regula sus actuaciones; así como en la institucionalidad del Estado que autoriza y vigila su funcionamiento ${ }^{16}$.

No obstante, para la comunidad, existen "vicios de legitimidad" ${ }^{17}$ en estos procederes. La legitimidad para la población cajamarcuna no se limita al cumplimiento de las normativas legales, sino a la correspondencia de estas con los derechos humanos. Ven en el actuar, tanto de la empresa como del Estado, un desconocimiento de los derechos humanos y de los valores identitarios sobre su territorio, así todas esas actuaciones se hagan en el marco de la legalidad. Legalidad que se ve deslegitimada por la población.

Hasta ahora, el conflicto pareciera presentarse desde las diferentes percepciones de las partes frente a la norma, el objeto de poder y las distintas dimensiones sociales, económicas y políticas. Sin embargo, el conflicto va más allá del punto de vista de las partes, toda vez que "cada actor sigue una racionalidad y define el espacio del conflicto, conforme a criterios propios" (Fontaine, 2004, p. 525). La confrontación de las partes radica en la perspectiva de legitimidad o deslegitimidad, si también así quiere comprenderse. La legitimación depende del nivel de percepción que la comunidad tiene frente al derecho, en la medida en que este reconoce sus costumbres, sus valores, territorios y aun sus justicias. Del grado de percepciones sobre estos factores depende el reconocimiento o no del derecho, o más bien dicho, de la legitimidad de este.

[...] los pobladores desconocen al derecho debido a que sus normas entran en conflicto con otro tipo de normas sociales o de costumbres que ellos consideran que son tan o más importantes que el derecho mismo, cuando les toca escoger entre la ley y tales costumbres, escogen estas últimas. (García, 2004b, p. 127)

La consideración de normas jurídicas como no jurídicas constituye un elemento fundamental para su legitimidad, esto es, como se ha descrito anteriormente, de su 
aceptación por parte de la comunidad. En este sentido, debería hacerse una distinción entre la legitimidad de las normas y la legitimidad del derecho. Este último permitiría la comprensión y consideración de los aspectos no solo normativos e institucionales que, en palabras de Weber (1944), representan los poderes de dominación. Dentro de este concepto también se comprenden las normas sociales, las costumbres, las ideologías de los territorios y todos los aspectos asociados con los valores de la comunidad. Finalmente, es desde y por estos frentes que subyacen los conflictos sociales, que para el caso objeto de estudio serían los conflictos socioambientales.

\section{CONCLUSIONES}

Los conflictos como parte de los procesos propios de las interacciones humanas están envueltos de características no solo sociales, sino también políticas y jurídicas. Estos aspectos involucran diferentes factores de juicio, valoraciones y oposiciones que dan lugar al conflicto, esto es, al enfrentamiento de estructuras e interacciones, producto de la confrontación de intereses y poderes. En este caso, se denota que las políticas económicas y sociales del Estado parecen estar en contravía de las situaciones y problemáticas de los territorios; de un lado, el plan de desarrollo nacional no solo legitima sino que además potencializa los procesos de exploración y explotación minera, mientras que en las zonas donde se realizan estos procesos se percibe por parte de la comunidad una afectación a su entorno natural y a su cultura, costumbres y tradición productiva que provoca una alteración a sus estilos de vida e identidades.

En este sentido, los conflictos tienen partes, representadas en actores y en sujetos, las cuales obedeciendo a sus propias percepciones entran en defensa de sus propios postulados. Ahora bien, esos postulados no necesariamente acampan en el plano de la ilegalidad, como podría creerse desde la teoría del derecho. Tanto los individuos como las estructuras que hacen parte del conflicto argumentan factores legales y legítimos en su defensa. Esa legitimidad, concepto polisémico en los estudios jurídicos, posee un alcance amplio en sus interpretaciones, que agotan el discurso de la validez y eficacia del derecho y que propenden por la comprensión de su definición en contextos más sociales -más allá de su propia definición jurídica-.

Es en esta perspectiva en que se problematizó el proyecto minero objeto de conflicto y objeto de estudio. En este escenario confluyen diferentes actores que defienden su legitimidad. Unos desde la legitimidad de la norma y de sus instituciones y otros desde la legitimidad de sus valores, cultura e ideologías. Ambos aspectos cobijados y amparados por las normas, claro está, tanto normas jurídicas como sociales. Pero tanto las unas como las otras componen el derecho. Así es, aunque pueda sonar escandaloso para la teoría clásica del derecho, las normas sociales también legitiman las normas jurídicas; finalmente, en la mayoría de los casos, las normas jurídicas alcanzan su nivel de aceptación en la medida en que guarden correspondencia con sus normas sociales. ¿Todas? Por supuesto que no. Son aquellas normas que encuentran identidades con los derechos de la comunidad las que alcanzan ese nivel de legitimidad, que no es lo mismo que cumplimiento.

Mientras el Estado se legitime solo por el poder que le confieren las normas y con ellas la coacción, podrá ser un Estado con normas válidas y tal vez eficaces. No obstante, estos dos últimos aspectos no garantizan su legitimidad, y mientras este último aspecto no sea reconocido por las comunidades los conflictos seguirán subyaciendo y 
las luchas y confrontaciones seguirán deslegitimando a las normas y a la institucionalidad.

\section{REFERENCIAS}

- Alarcón, G. (2011). Estado de derecho, derechos humanos y democracia: Pautas para la racionalidad jurídico-política desde Elías Díaz. España: Librería-Editorial Dykinson.

- AngloGold Ashanti. (2014). Nuestra posición: informe de Colombia Solidarity Campaign. Colombia.

- AngloGold Ashanti. (s.f.). La Colosa. Una oportunidad de oro para el Tolima. Recuperado de Link

- Colombia Solidarity Campaign. (2011). La Colosa: una muerte anunciada. Colombia.

- Concejo Municipal. Decreto 073 de 2000. Esquema de Ordenamiento Territorial. Cajamarca-Tolima. Colombia.

- Congreso de Colombia (15 de Agosto de 2001) Código de Minas (Ley 685 de 2001).

- Constitución Política de 1991. Colombia: Editorial Leyer.

- Coser, L. A. (1961). Las funciones del conflicto social. México: Fondo de Cultura Económica.

- Departamento Nacional de Planeación. Plan Nacional de Desarrollo 2010-2014. Colombia.

- Durán, P. (1997). Notas de Teoría del Derecho. España: Universidad Jume I Castello.

- Felstiner, W., Abel, R., \& Sarat, A. (2001). Origen y trasformación de los conflictos: reconocimiento, acusación, reclamación... En M. García Villegas (Ed.),Sociología jurídica. Bogotá: Universidad Nacional de Colombia.

- Fontaine, G. (2004). Enfoques conceptuales y metodológicos para una sociología de los conflictos ambientales. En Guerra, sociedad y medio ambiente. Foro Nacional Ambiental. Recuperado de Link

- Garay, L. (2013). Economía ecológica, ecología política y justicia ambiental, y neoinstitucionalismo. Algunas aproximaciones para el análisis de las problemáticas 
alrededor de la explotación de recursos naturales no renovables. EnMinería en Colombia. Institucionalidad y territorio, paradojas y conflictos. Colombia: Contraloría General de la República.

- García Muñoz, C. (2014a). Desplazamiento ambiental: polisemias y tensiones de una categoría emergente. Revista DELOS: Desarrollo Local Sostenible, 7(20).

- García Villegas, M. (2014b). La eficacia simbólica del Derecho. Sociología política del campo jurídico en América Latina. Segunda edición. Colombia: IEPRI.

- Leff, E. (2003). La ecología política en América Latina. Un campo de construcción.Revista Sociedade e Estado, 18(1-2), 17-40.

- Martínez, J., \& Roca, J. (2001). Economía ecológica y política ambiental. Segunda edición. México: Fondo de Cultura Económica.

- Martínez-Alier, J. (2006). Los conflictos ecológico-distributivos y los indicadores de sustentabilidad. Polis, Revista de la Universidad Bolivariana, 5(13).

- Pérez, H., Zárate, C., \& Turbay, S. (2011). Conflictos ambientales: la biodiversidad como estrategia ordenadora del territorio. Opinión Jurídica, 10, 89-104.

- Plan de Desarrollo Municipal de Cajamarca (2012-2015) "Para volver a creer". Colombia.

- Rubio, I. (2014). Propiedad y naturaleza en la configuración socioambiental de los conflictos. A propósito de los parque eólicos en el istmo de Tehuantepec. EnConflictos, conflictividades y movilizaciones socio-ambientales en México. Problemas comunes y lecturas diversas. México: Universidad Autónoma de México.

- Silva, G. (2008). La teoría del conflicto. Un marco teórico necesario.Prolegómenos. Derechos y Valores, $\mathrm{XI}(22), 29-43$.

- Weber, M. (1944). Economía y sociedad. México: Fondo de Cultura Económica.

- Weber, M. (2006). Estructuras de poder. México: Ediciones Coyoacán.

1. El artículo se deriva del proyecto de investigación titulado: "Conflictos y movimientos sociales de jóvenes en contextos de vulnerabilidad en la eco-región eje cafetero: hacía un proceso de constitucionalismo popular". Financiado por la Universidad de Manizales, el cual hace parte del programa de investigación "Sentidos y prácticas políticas de niños, niñas y jóvenes en contextos de vulnerabilidad en el eje cafetero, 
Antioquía y Bogotá: un camino posible de consolidación de la democracia, la paz y la reconciliación mediante procesos de formación ciudadana". Este programa está conformado por 3 instituciones: CINDE, Universidad de Manizales y Universidad Pedagógica Nacional, aprobado por Colciencias en el marco de la Convocatoria Pública No. 542 de 2011.

2. Abogada. Magíster en Desarrollo Sostenible y Medio Ambiente. Estudiante Doctorado en Desarrollo Sostenible Universidad de Manizales. Docente-Investigadora, Universidad de Manizales. Manizales, Colombia.cmunevar@umanizales.edu.co. ORCID iD 0000-0002-9190-6232

3. Abogada. Estudiante de Maestría en Desarrollo Sostenible y Medio Ambiente, Universidad de Manizales - Asistente de Investigación, Semillero de Investigación en Derecho y Conflictos Ambientales. Manizales, Colombia luisaf0410@hotmail.com. ORCID iD 0000-0002-0056-1743

4. Abogada. Estudiante de Maestría en Desarrollo Sostenible y Medio Ambiente, Universidad de Manizales - Asistente de Investigación, Semillero de Investigación en Derecho y Conflictos Ambientales. Manizales, Colombia.anyelaahenao@hotmail.com. ORCID iD 0000-0002-7206-755X

5. Desde un enfoque constructivista Felstiner, Abel \& Sarat (2001, p. 41), definen: "Los conflictos no son cosas, son construcciones sociales".

6. Sobre el alcance de lo socioambiental Rubio (2014, p. 126) establece que: "Es preciso entender que la palabra socio ambiental debería ser más que la unión de dos o más términos que en la práctica se muestran separados. Al margen de preservar los espacios disciplinares que son fundamentales para la producción de datos e información, lo socio ambiental apunta a una dimensión común, un espacio de sentido que puede estar presente, o no, en una disputa por el territorio o algún recurso".

\section{AngloGold Ashanti (s.f.). La Colosa. Una oportunidad de oro para el Tolima.}

8. Según el informe de Colombia Solidarity Campaign (2011): "Cajamarca es un distribuidor importante de productos agrícolas para todo el país, siendo la despensa agrícola de Colombia; su clima templado es propicio para cultivos tales como la arracacha, el café, judías, guisantes, maíz, pimiento y muchas variedades de frutas tales como naranja, plátano y banano. También cuenta con fuentes de ríos que contribuyen de forma fundamental al regadío de las cosechas en otros lugares del departamento".

9. 'Los economistas suelen llamar 'externalidades' a los daños causados por una actividad cuyo valor no viene recogido en los costos y precios establecidos en los mercados" (Martínez \& Roca, 2001, p. 447).

10. Ver objetivo 5. Esquema de Ordenamiento Territorial de Cajamarca, año 2000.

11. Artículo 80: "El Estado planificará el manejo y aprovechamiento de los recursos naturales, para garantizar su desarrollo sostenible, su conservación, restauración o sustitución. Además, deberá prevenir y controlar los factores de deterioro ambiental, imponer las sanciones legales y exigir la reparación de los daños causados [...]".

12. Plan Nacional de Desarrollo (p. 205): "Las locomotoras de crecimiento son los sectores o actividades económicas que van a definir el rumbo que tome la economía colombiana en los próximos años. Sectores con el potencial de mejorar continuamente 
el uso y la combinación eficiente de factores como el capital, el trabajo o los recursos naturales".

13. Plan Nacional de Desarrollo (p. 205). "Las cinco locomotoras de crecimiento identificadas por el gobierno [...] son: 1) nuevos sectores basados en la innovación, 2) agricultura y desarrollo rural, 3) vivienda y ciudades amables, 4) desarrollo minero y expansión enérgica, 5) infraestructura de transporte".

14. Informe de AngloGold Ashanti (2014, p. 15): "En el año 2011, INGEOMINAS otorgó a nuestra compañía en Cajamarca, Tolima, los contratos de concesión minera EIG163, GGF-151 y GLN-09261X, ubicados en la zona en la cual se está desarrollando el Proyecto exploratorio la Colosa. Otorgadas las concesiones mineras, acudimos al Ministerio Amiente para recibir orientación sobre los trámites a seguir, y las indicaciones de interacción con CORTOLIMA durante toda la fase de exploración; pues al no requerir licencia ambiental para la fase exploratoria, en ella recaía la competencia para el seguimiento de las actividades".

15. García (2014b, p. 60) acuña el término de eficacia simbólica como: "una forma de captar la lógica de las luchas políticas que, en buena parte, son luchas por el sentido de las palabras".

16. En pronunciamiento de la multinacional, sus actuaciones se encuentran legitimadas en el marco de la legalidad, vigilado y supervisado por la autoridad ambiental, en este caso, la Corporación Autónoma Regional del Tolima, CORTOLIMA: "Desde el 2010 contamos con la certificación en el sistema de gestión de seguridad industrial y salud ocupacional OHSAS 18001.2007; y cumplimos con todos los estándares nacionales e internacionales para el transporte y almacenamiento de sustancias químicas. Todas las sustancias químicas que se usan en el Proyecto la Colosa, cuentan con sus respectivas hojas de seguridad y son almacenadas, transportadas y manipuladas acorde con la normatividad vigente y las normas técnicas obligatorias. Esto ha sido evidenciado por CORTOLIMA" (AngloGold Ashanti, 2014, p. 8).

17. Esta expresión fue utilizada por un ponente en la audiencia pública por el Congreso de la República de Colombia en el municipio de Cajamarca (Tolima), para referirse a un Estado que en el marco de sus actuaciones no garantiza los derechos humanos.

Para citar este artículo: Munévar Quintero, C. A., González Londoño, L. F., \& Henao Londoño, A. A. (2017). Conflictos socioambientales: entre la legitimidad normativa y las legitimidades sociales. Caso mina La Colosa, Cajamarca (Tolima, Colombia). Luna Azul, 44, 165-176. DOI: 10.17151/luaz.2017.44.10. Recuperado de http://200.21.104.25/lunazul/index.php/component/content/article?id=226

Esta obra está bajo una Licencia de Creative Commons Reconocimiento CC BY

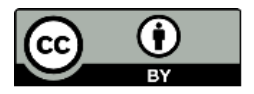

